\title{
Transplantation of NGF-Gene-Modified Bone Marrow Stromal Cells into a Rat Model of Alzheimer' Disease
}

\author{
Li-Yan Li • Jin-Tao Li • Qing-Ying Wu • Jin Li • \\ Zhong-Tang Feng $\cdot$ Su Liu $\cdot$ Ting-Hua Wang
}

Published online: 19 March 2008

(C) Humana Press Inc. 2008

Erratum to: J Mol Neurosci

DOI 10.1007/s12031-007-9022-x

Figure 2 is hereby replaced with the following:
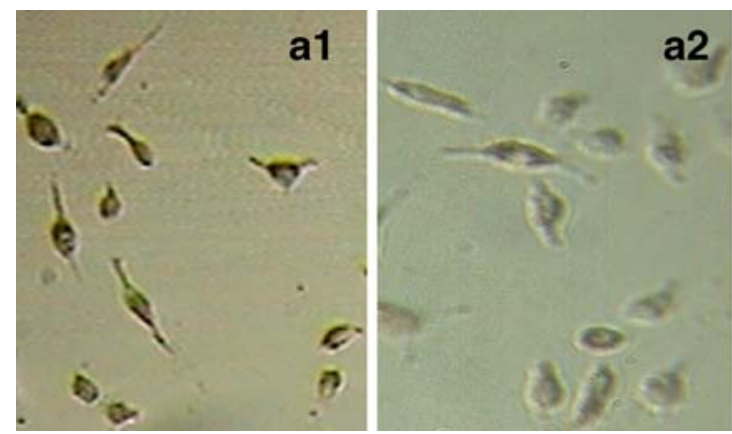

Figure 2 a Anti-NGF on BMSC transfected with recombinant (a1) and control (a2). BMSCs expressing NGF were seen in the transfected NGF gene group (a1), but not in non-transfected NGF gene group

Figure 4 and its legend are hereby replaced with the following:

The online version of the original article can be found at http://dx.doi. org/10.1007/s12031-007-9022-x

L.-Y. Li · J.-T. Li • Q.-Y. Wu J. Li $\cdot$ Z.-T. Feng $\cdot$ S. Liu •

T.-H. Wang $(\bowtie)$

Institute of Neuroscience, Kunming Medical College,

Kunming, Yunnan Province 650031, China

e-mail: tinghua_neuron@263.net

\section{S. Liu $\cdot$ T.-H. Wang}

Department of Histology, Embryology and Neurobiology,

West China School of Preclinical and Forensic Medicine,

Sichuan University,

Chengdu 610041, China
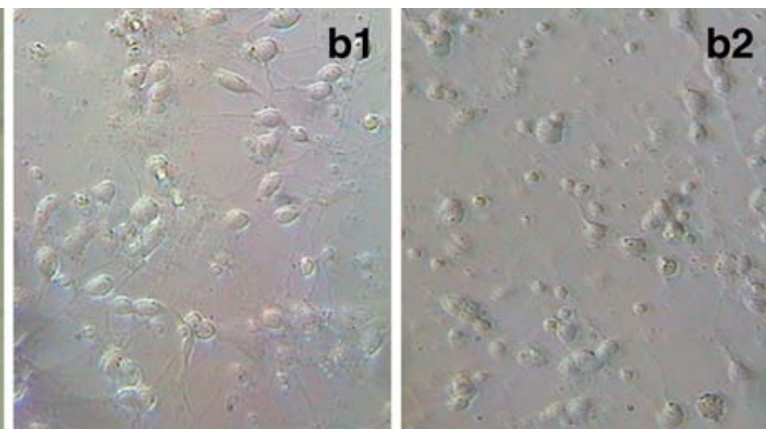

(a2). b Extension-like processes of neurons in the supernatant of NGF-gene-modified BMSC (b1) shows more significant than in the control group (b2). a and $\mathbf{b}$ Inverted phase contrast microscope $\times 250$

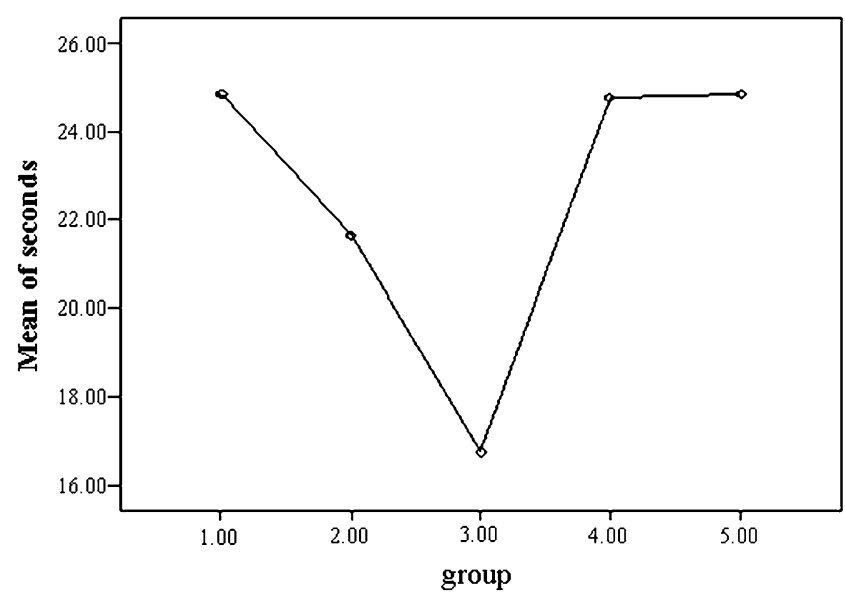

Figure 4 The results from the test for space exploration showed that the swim tracks of rats in group 4 mostly reached the underwater position of the platform but not those in the AD group. A significant decline in cognitive ability was observed in the $\mathrm{AD}$ group, while a significant improvement in cognition was seen in the BMSC or BMSC-NGF treated groups 